HANSEN, M. E. \& MCINTIRE D. D. (1994) Reference citations in radiology: accuracy and appropriateness of use in two major journals. American Journal of Roentgenology. 163, 719-723.

*L. A. Lawson, Senior Registrar in Forensic Psychiatry, Regional Secure Unit, Runwell
Hospital, The Chase, Wickford, Essex SS11 7QE; and Ruth Fosker, District Librarian, Colchester and North East Essex Postgraduate Medical Centre, Colchester General Hospital, Colchester.

\title{
The management of cylindrical battery ingestion in psychiatric settings
}

\author{
Nick Hindley, Harvey Gordon, Chris Newrith and Damian Mohan
}

\begin{abstract}
Aim and method To describe physical sequelae of cylindrical battery ingestion and their management, by description of recent cases and literature review. Results X-rays should be performed to determine the position and integrity of the battery. In the absence of abdominal symptoms, immediate surgical opinion may not be indicated.

Clinical implications Conservative management of battery swallowing is frequently possible.
\end{abstract}

There have been several recent episodes involving the swallowing of cylindrical batteries on the intensive care and admissions units at Broadmoor Hospital. People resident in these units have access to personal stereos which in almost all cases have been the source of the batteries which were subsequently swallowed. All episodes appear to have occurred at times of acute psychiatric disturbance and would seem to fit into the category of deliberate selfharm rather than frank suicidal intent. This particular behaviour appeared to follow a vogue over a period of two to three months before waning.

From the medical point of view, even psychiatrists well-acquainted with deliberate selfharm may be unfamiliar with the management of battery ingestion. Ingestion of foreign objects can create considerable anxieties and it can be that considerable pressure is put on the attending doctor to 'do something'. In such situations, prior knowledge of the potential sequelae of the act together with a clear picture of what reasonably needs to be done to ensure the person's safety can do much to diffuse such anxieties and to minimise the disruption caused both to and by the individual concerned.

The incidents encountered at Broadmoor provide some indication of the range of sequelae of battery swallowing and the extent to which management may vary according to the particular situation.

\section{Case illustrations}

\section{Patient 1}

A man in his twenties with a diagnosis of personality disorder and a long history of severe self-harm including self-laceration and persistent head-banging. During a period of acute disturbance he swallowed an alkaline cylindrical battery having bitten the casing to allow release of its contents. He became immediately symptomatic with acute upper gastrointestinal pain. Abdominal X-ray (AXR) showed a damaged battery in the stomach. He was treated as a surgical emergency and underwent laparotomy with removal of the battery. On return to Broadmoor he swallowed two further batteries in similar fashion resulting in a repeat laparotomy; 
this second operation was followed by Clostridium difficile infection.

\section{Patient 2}

A man in his late teenage years with a diagnosis of psychotic illness and personality disorder. He swallowed two 'live' cylindrical alkaline batteries. There was no history of damage to the battery casing and no physical symptoms. AXR showed undamaged batteries beyond the pylorus. The batteries were passed per rectum a week later.

\section{Patient 3}

A man in his late twenties with a diagnosis of depressive disorder with psychotic exacerbations and personality disorder with a long history of self-mutilation and serious suicide attempts. He attempted to damage the cases of two alkaline batteries with his teeth before swallowing them. No acute symptoms followed ingestion. AXR confirmed batteries with damaged casing beyond the pylorus. There were no clinical symptoms suggesting leakage and the batteries passed per rectum within two weeks.

\section{Patient 4}

A man in his twenties with a diagnosis of psychotic illness, personality disorder and mild learning difficulties and a history of almost unremitting self-harm with severe headbanging. He swallowed two spent alkaline batteries without subsequent associated symptoms. AXR demonstrated batteries beyond the pylorus and the batteries were passed per rectum within two weeks.

\section{Patient 5}

A man in his thirties with a diagnosis of personality disorder with psychotic exacerbations and an extended history of self-harm. He also had a long history of constipation. He swallowed four alkaline cylindrical batteries during a period of depressed mood. All the batteries were noted to have passed the pylorus on AXR. Three batteries were passed per rectum between one and two months after ingestion; a fourth remained in the caecum after three months. There was no evidence of damage to the battery casing noted on recent AXR. The surgical team recommend conservative management.

\section{Discussion}

The majority of the literature concerning battery ingestion deals with button batteries (as found in hearing aids and watches) rather than with the cylindrical batteries found in personal stereos (further details available from the author upon request). Button batteries it would seem, are more likely to be swallowed either by accident or out of curiosity by a young child (David \& Ferguson, 1986). It would seem furthermore that, given their greater size, cylindrical batteries are largely ingested only by those intending to do so. Individuals swallowing cylindrical batteries were greatly outnumbered by those ingesting button cells (62 v. 2382) in a seven-year followup study from the National Capital Poison Center in Washington DC (Litowitz \& Schmitz, 1992). The majority of all batteries (cylindrical included) pass through the gastrointestinal tract uneventfully. Only rarely do batteries appear to become lodged in the gastrointestinal tract; however when this occurs, particularly in the oesophagus or stomach some form of operative intervention may be needed.

Cylindrical cells are typically alkaline-manganese or nickel-cadmium (rechargeable) and when ingested are capable of causing both corrosive and toxic damage. Such damage would occur if the integrity of the battery casing was deliberately damaged (as in Patient 1) or as a result of sustained acid attack from gastric contents over a period of weeks rather than days. Alkaline battery contents can cause severe caustic injury and in theory nickel and cadmium can have significant systemic effects.

Electrical burns from live batteries are apparently much less likely with cylindrical batteries than with the button variety. In their study Litovitz \& Schmitz (1992) noted no significant effect on outcome exercised by battery discharge state (i.e. whether live or spent) and this may be attributable to the effect of the gastric juices discharging of live cells.

It would thus seem that major complications following cylindrical battery ingestion relate to the battery's loss of integrity. Clinical symptoms relating to such an event may be delayed, but are usually unequivocal and require swift clinical response when they occur. In the absence of clinical symptoms, the National Poisons Information Service (personal communication, 1998) recommend repeat $A X R s$ to determine the position and condition of the ingested battery - any damage to the battery casing can be seen on a plain film. It is recommended that such investigations should be performed at presentation. after 24 hours (to ensure passage beyond the pylorus) and then at 48-hour intervals. A surgical opinion with a view to surgical removal should be sought if:

(i) the battery was damaged before or during ingestion especially if there are clinical signs or symptoms of corrosive damage:

(ii) the battery remains in the stomach 24 hours post-ingestion;

(iii) there is no movement within a 48-hour period; 
(iv) there are clinical signs of leakage while the battery is in the gastrointestinal tract;

(v) there are any gastrointestinal effects, particularly gastroenteritis or discoloured stools.

Surgical interventions include laparotomy and removal of batteries in emergency situations and endoscopic removal of batteries in situations where there is a strong possibility of physical sequelae (for example, when the battery casing is seriously damaged or when the battery becomes lodged in the oesophagus or stomach). Litovitz \& Schmitz report that, over the seven-year period of their study, the use of endoscopic and surgical intervention declined more than fivefold.

In relation to the management of the five patients described above, the guidelines set out by the National Poisons Information Service were broadly adhered to. The importance of clinical symptoms as an indicator of the need for surgical intervention is emphasised. The case of Patient 3 demonstrates that conservative management with close clinical monitoring is possible within a psychiatric hospital in cases where there is radiological evidence of damage to the battery casing, provided that there are no abdominal symptoms and that liaison with the local surgical team is established. After the first two AXRs (at presentation and at 24 hours) in all cases in which there were no acute abdominal symptoms, subsequent radiological investigations were repeated on a weekly basis. While Patient 5 was somewhat unusual in the extended transit time of his remaining battery, there seems to be no indication for surgical intervention at the time of writing.

\section{Conclusion}

It would appear, both from our experience and from the literature, that cylindrical battery swallowing not accompanied by abdominal symptoms can be managed conservatively with the help of a radiological opinion and, if needed, telephone liaison with the local surgical team. It may also be that the need for immediate AXR at the time of presentation is not as pressing in situations of simple swallowing as it is in situations where there are abdominal symptoms or a history of deliberate damage to the battery prior to ingestion. Such considerations mean for the most part that visits to the general hospital by individuals who swallow batteries who are psychiatric in-patients need only be short and infrequent: this clearly is important where the risks of a patient absconding are high and where significant nursing resources may be needed to provide an escort.

Recent experience of battery swallowing at Broadmoor Hospital would suggest that the behaviour is adopted by people either wishing to harm themselves or consciously attempting to manipulate their subsequent management. A low key, yet informed response to asymptomatic individuals who swallow batteries will mean that any disruption to a carefully boundaried careplan can be kept to a minimum.

\section{References}

DAvid. T. J. \& FERGuson, A. P. (1986) The management of children who have swallowed button batteries. Archives of Disease in Childhood, 61, 321-322.

LrTowrTz. T. \& SchMrTz, B. F. (1992) Ingestion of cylindrical and button batteries: an analysis of 2382 cases. Pediatrics. 89 (suppl.), 747-757.

*Nick Hindley, Registrar in Psychiatry. Harvey Gordon, Consultant Forensic Psychiatrist, Chris Newrith, Specialist Registrar in Psychotherapy. Broadmoor Hospital, Crowthorne, Berkshire RG45 7EG; and Damian Mohan. Lecturer/ Honorary Senior Registrar in Forensic Psychiatry, University of Southampton and Broadmoor Hospital

*Correspondence 